\title{
Significance of Fundamental Diagrams to First-Order Macroscopic Traffic Modelling
}

\author{
Konstantinos Gkiotsalitis and Andy Chow \\ Centre for Transport Studies, University College London \\ gkiotsalitis@gmail.com
}

\begin{abstract}
Macroscopic models are computationally effective and generate reliable estimates of traffic dynamics based upon the principles of flow conservation and propagation, in which the underlying fundamental diagram (i.e. flow-density relationship) has an important part in capturing the plausible traffic propagation over time and space. This study investigates in practice whether, and in what extent, the calibration of the fundamental diagram affects the performance of macroscopic traffic modelling by using motorway data. For study purposes, a modified version of the first-order Cell Transmission Model (CTM) is adopted in order to allow the use of different fundamental diagrams instead of the traditional triangular one. Traffic data from the MIDAS (Motorway Incident Detection and Automatic Signalling) dataset for M25 motorway in the UK are used for the case studies. The findings of this study demonstrate the significance of selecting the best-suited fundamental diagram to each traffic scenario in order to improve the accuracy of traffic modelling and contribute to the performance analysis and management of motorways.
\end{abstract}

Keywords: Macroscopic traffic simulation, First-Order Models, Fundamental Diagram, Capacity Drop, MIDAS

\section{Introduction}

Traffic engineers and scientists use micro or macroscopic traffic models to estimate the evolution of traffic flow since it is a necessity for motorway management and traffic light settings [1]. Microscopic models [2-4] emulate the behavior of each driver/vehicle by drawing laws through the calibration of measurements from in-vehicle devices [5], but are computationally expensive.

Macroscopic models require less computation and describe the traffic flow dynamics through aggregated quantities such as volume, density and mean speed. The underpinning theory of a plethora of macroscopic models is derived from the hydrodynamic theory of traffic evolution [6] and extensions of it led to different versions of first-order models [7, 8]. In general, macroscopic models use a set of traffic parameters (traffic flow, free flow speed, congested wave speed, capacity, critical density and more) as data input, and their values are estimated during the calibration of the fundamental diagram by using field data from motorways.

The underlying fundamental diagram (i.e., the steady state flow-density relationship) is the crux of macroscopic models for capturing the traffic dynamics including the formation and dissipation of congestion, evolution of queue size and journey times (see examples in [9], [10]). A comprehensive review of different forms of fundamental diagrams can be found in [11]. In addition, apart from different forms of fundamental diagrams, a number of calibration algorithms have also been proposed for the estimation of traffic parameters [12-16]. 
Different fundamental diagrams and associated calibration methods are expected to generate dissimilar traffic parameter values and affect the estimation of traffic dynamics. This paper investigates the significance of fundamental diagrams to macroscopic traffic modelling and is organized as follows: in Section 2, a revised CTM that enables the use of different fundamental diagrams is presented. In Section 3, three different fundamental diagram calibration methods are discussed. Section 4 presents a case study where traffic data collected from M25 motorway in the UK are adopted to analyses the variation in accuracy of first-order models under the use of different fundamental diagrams. Section 5 provides some concluding remarks.

\section{Cell Transmission Model}

The first-order kinematic wave model [1] assumes that the speed adjusts to the corresponding traffic density instantly and the flow-density evolution is described by the conservation law:

$$
\frac{\partial \mathrm{k}(\mathrm{x}, \mathrm{t})}{\partial \mathrm{t}}+\frac{\partial \mathrm{f}(\mathrm{x}, \mathrm{t})}{\partial \mathrm{x}}=\mathrm{r}(\mathrm{x}, \mathrm{t})-\mathrm{s}(\mathrm{x}, \mathrm{t})
$$

where $k(x, t), f(x, t)$ are the average flow and density at location $x$ at time $t$ and $r(x, t)$ and $\mathrm{s}(\mathrm{x}, \mathrm{t})$ are the inflow and outflow at location $\mathrm{x}$ and time $\mathrm{t}$.

One of the most efficient discretization methods for solving the kinematic wave model is the Cell Transmission Model (CTM) [7]. The CTM updates dynamically only the evolution of the number of vehicles per road segment (cell) along a motorway model). CTM predicts the traffic behavior within each cell by splitting the entire network that includes a number of purpose-built cells (CTM is a discrete equivalent of the classical hydrodynamic to a finite number of carefully selected intermediate points, and is a reliable, simple and well-tested model of freeway traffic. Due to its simplicity and plausibility, CTM underlies a number of transport system modelling and management tools $[8,17]$.

The importance of simple and accurate macroscopic models (such as first-order models and especially the CTM which is robust and matches the results of hydrodynamic theory even for large cell sizes) was demonstrated in the work of [8]. Nevertheless, discretized models generate spatio-temporal errors during the estimation of traffic evolution which are usually comparable to the selected time interval and the cell size. In addition, discretized models fail to represent accurately the form of the wave shocks caused by congestion.

In this study we use a modified version of the first-order CTM to describe the evolution of traffic over a one-way road segment by updating the traffic conditions with each tick of the simulation clock (simulation time). The simulation time step, dt, is selected in order to ensure that vehicles do not advance over two cells within one tick of the simulation clock under light traffic: 


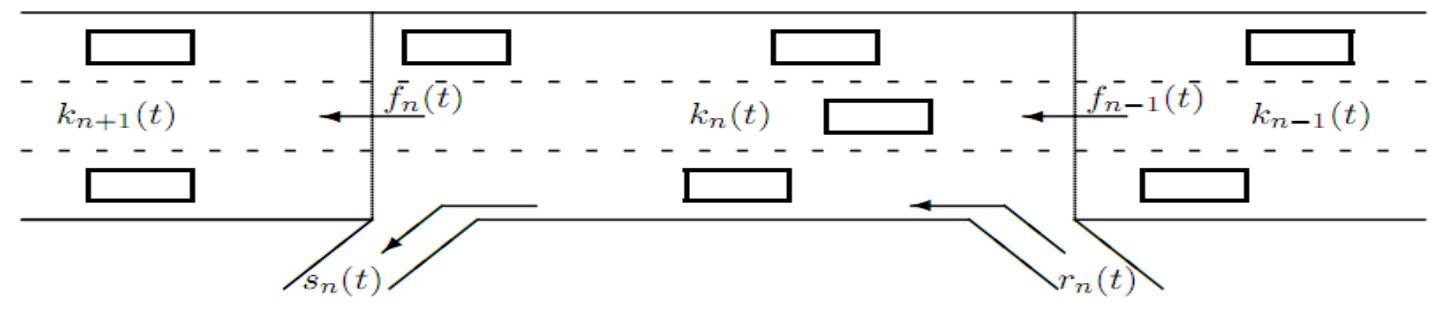

Figure 1. Flow Conservation within a Cell $-r n(t)$ : on-Ramp Flow, $\operatorname{sn}(t)$ : Off-ramp Flow

$$
\mathrm{dt} \leq \min \frac{\Delta \mathrm{x}_{\mathrm{n}}}{\mathrm{v}_{\mathrm{n}}}, \forall \mathrm{n}
$$

, where $\Delta x_{n}$ is the length of cell $n$ and $v_{n}$ is the free flow speed of cell $n$ (derived from the calibration of the fundamental diagram).

Each cell $\mathrm{n}$ represents a road segment and has a number of attributes (i.e., length, number of lanes). In a more general case, where a cell $n$ has one on-ramp and one off-ramp, the flow conservation within the cell is:

$$
\mathrm{k}_{\mathrm{n}}(\mathrm{t}+1)=\mathrm{k}_{\mathrm{n}}(\mathrm{t})+\left(\mathrm{f}_{\mathrm{n}-1}(\mathrm{t})+\mathrm{r}_{\mathrm{n}}(\mathrm{t})-\mathrm{f}_{\mathrm{n}}(\mathrm{t})-\mathrm{s}_{\mathrm{n}}(\mathrm{t})\right) \frac{\Delta \mathrm{x}_{\mathrm{n}}}{\mathrm{dt}}
$$

, $\Delta \mathrm{x}_{\mathrm{n}}$ is the length andk $\mathrm{k}_{\mathrm{n}}(\mathrm{t})$ the traffic density of cell $\mathrm{n}$ at time $\mathrm{t}, \mathrm{f}_{\mathrm{n}}(\mathrm{t})$ is the outflow from cell $n$ and $f_{n-1}(t)$ is the inflow to cell $n$ in vehicles per hour, $r_{n}(t)$ is the on-ramp flow and $s_{n}(t)$ is the off-ramp flow in vehicles per hour.

In free flow conditions $f_{n}(t)=v_{n} k_{n}(t)-s_{n}(t)$, where $v_{n}$ is the free flow speed of cell $n$. Furthermore, if the on-ramp flow is not measured by on-ramp detectors, then $s_{i}(t)$ is often given by split ratios: $s_{n}(t)=b_{n}\left(s_{n}(t)+f_{n}(t)\right)$, where $b_{n}$ is a constant value.

The outflow $f_{n}(t)$ from cell $n$ at time $t$ is computed by using the values of the estimated traffic parameters from the calibration phase. The outflow $f_{n}(t)$ is equal to the minimum value of the three following quantities:

$$
\left.f_{n}(t)=\min \left(v_{n} k_{n}(t)-s_{n}(t) ; Q_{n} ; w_{n+1}\left(k_{j a m, n+1}-k_{n+1}(t)\right)\right)\right)
$$

, where $v_{n} k_{n}(t)-s_{n}(t)$ is known as the traffic demand, $Q_{n}$ is the capacity of cell $n$ and $w_{n+1}\left(k_{j a m, n+1}-k_{n+1}(t)\right)$ is the supply. The required parameters: ( $v_{n}$ : free flow speed, $Q_{n}$ : capacity, $w_{n+1}$ : congested speed and kjam, $n+1$ : jam density) are estimated from the calibration of the fundamental diagrams of cells $n$ and $n+1$ respectively before the implementation of the CTM model.

The CTM can be expanded later on to cover all cells in the network. The traffic data, which is required for the implementation of the model, is provided at Table 1:

Finally, due to lack of inductive loop or other traffic detectors or other at the off-ramps, the off-ramp flow, $s_{n}(t)$, can be derived from an estimation of the portion of the flow which is directed to another link and not from observed traffic data. Hence, a non-negative matrix $\beta_{\mathrm{nj}}(\mathrm{t})$ ) is assigned to define empirically the portion of the vehicle flow approaching from input link $\mathrm{n}$ and directed to the output link $\mathrm{j}$ at time $\mathrm{t}$.

Apart from the classic CTM, versions of first-order models for traffic simulation are presented by $[8,12]$ and [18]. The first-order mathematic model which is used in this study 
Table 1. Data Requirements

\begin{tabular}{|c|l|}
\hline Boundary Conditions & $\begin{array}{l}\text { The inow fO(t) entering the _rst cell for each tick } \\
\text { of the simulation clock } \\
\text { The on-ramp ows rn }(\mathrm{t}) \text { for each tick of the } \\
\text { simulation clock } \\
\text { The o_-ramp ows sn }(\mathrm{t}) \text { for each tick of the } \\
\text { simulation clock }\end{array}$ \\
\hline Initialization & $\begin{array}{l}\text { The initial densities on the freeway, kn }(\mathrm{t} 0), \text { which } \\
\text { are derived from station measurements for time t0 }\end{array}$ \\
\hline
\end{tabular}

to capture the traffic evolution is described below.

1. Consider a motorway section divided into $I=\{1, \ldots, N\}$ consequent cells, where each cell, $\mathrm{n} \in \mathrm{I}$ has a length $\Delta \mathrm{x}_{n} \in \Re^{+}$

2. From the calibration of the fundamental diagram estimate the parameters $v_{n} ; \mathrm{I} \rightarrow \Re^{+}$ (free flow speed), $Q_{n} ; \mathrm{I} \rightarrow \Re^{+}$(cell's capacity), $k_{n_{\text {,crit }}} ; \mathrm{I} \rightarrow \Re^{+}$(critical density), $w_{n}$; $\mathrm{I} \rightarrow \Re^{+}$(congested speed), $k_{n, j a m} ; \mathrm{I} \rightarrow \Re^{+}$(jam density) for each cell $\mathrm{n} \in \mathrm{I}$.

3. Select a simulation time, dt, such that $\mathrm{dt} \leq \min \frac{\Delta x_{n}}{v_{n}}, \forall \mathrm{n} \in \mathrm{I}$. Then, divide the total time of the simulation into $\mathrm{T}=\left\{\mathrm{t}_{0}, \ldots, \mathrm{t}, \mathrm{t}+1, \ldots, \mathrm{t}_{v}\right\}$ discrete time steps, where the time step: $(\mathrm{t}+1)-\mathrm{t}=\mathrm{dt}$.

4. Derive from real traffic data measurements the inflow at cell $n=1, f_{0}(t), \forall \mathrm{t} \in \mathrm{T}$, the initial densities, $k_{n}\left(t_{0}\right), \forall \mathrm{n} \in \mathrm{I}$, the inflow demand from a ramp entering a cell $\mathrm{n}$, $R D_{n}(t), \forall \mathrm{n} \in \mathrm{I}, \forall \mathrm{t} \in \mathrm{T}$, and the outflows to a ramp exiting cell $\mathrm{n}, s_{n}(t) \forall \mathrm{n} \in \mathrm{I}, \mathrm{t} \in \mathrm{T}$.

5. For $\mathrm{t}=\mathrm{t}_{0}$ follow the steps $6-10$ :

6. Compute the capacity of each ramp entering at cell $n \in I$, Qramp,n. Compute the inflow at each cell $\mathrm{n} \in \mathrm{I}$ from on-ramps, $r_{i}(t)$,:

$$
r_{n}(t)=\min \left(Q_{\text {ramp.n. }} ; R D_{n}(t) ; \frac{R D_{n}(t)}{d t} ;\left(k_{j a m, n}-k_{n}\right) \frac{\Delta x_{n}}{d t}\right)
$$

, where $R Q_{i}(t)$ is the queue of the ramp inserting at cell $\mathrm{i}$ at time t.

7. Compute the evolution of the queue of the ramp inserting at cell $\mathrm{n}$ :

$$
\left.R Q_{n}(t+i)=\max \left(0 ; R Q_{n}(t)-r_{n}(t)\right) d t\right)
$$

8. Compute the traffic flow at each cell $n \in I$ :

$$
f_{n}(t)=\min \left(v_{n} k_{n}(t)-s_{n}(t) ; Q_{n} ; w_{n+1}\left(k_{j a m, n+1}-k_{n+1}(t)-\frac{r_{n+1}(t)}{\Delta x_{n+1}} d t\right)\right)
$$

, where, $\operatorname{Sn}(\mathrm{t})$, is the outflow from the outgoing ramp of cell $\mathrm{n}$ (derived by real time mea-surements). Moreover, the traffic flow for the $\operatorname{sink}($ cell $n=N$ ) is:

$$
f_{n}(t)=\min \left(v_{n} k_{n}(t)-s_{n}(t) ; Q_{n}\right)
$$

9. Compute the evolution of traffic density for cell $n=1$ :

$$
k_{1}(t+1)=k_{1}(t)-\left(r_{1}(t)-s_{1}(t)+f_{0}(t)-f_{1}(t)\right) \frac{d t}{\Delta x_{1}}
$$


10. Compute the evolution of traffic density for all other cells:

$$
k_{n}(t+1)=k_{n}(t)-\left(r_{n}(t)-s_{n}(t)+f_{n-1}(t)-f_{n}(t)\right) \frac{d t}{\Delta x_{n}}
$$

11. Until $\mathrm{t}=\mathrm{t} \mathrm{v}:$ update $\mathrm{t}=\mathrm{t}+1$ and follow the steps $6: 10$.

In this model, we assume that the vehicles are uniformly distributed over the cell, and all vehicles travel at the same average speed in order to satisfy the First-In, First-Out (FIFO) principle.

\section{Fundamental Diagrams}

The calibration of the fundamental diagram leads to the determination of various model parameters such as free-flow speed, capacity flow, and shockwave speed from detector data. Examples of calibration methods include least square estimation, Kernel Inversed Lambda $[13,14]$ and Quantile Regression [15]. Different calibration methods yield dif-ferent input for first-order models, and thus it is important to investigate the influence of calibration algorithms to the performance of first-order models. The remaining of this section is devoted to the description of three different fundamental diagrams. Since there are numerous different forms of fundamental diagrams [11], we selected three calibration methods for our comparative analysis which are scalable and able to calibrate automatically numerous diagrams without excessive computational requirements. In this section, the daily traffic observations of detector 5158A from motorway M25 (May 18th, 2011) 5158A [19] are adopted for an initial demonstration of the calibration methods (for a comprehensive description of the examined section of motorway M25 refer to Section 4).

\subsection{Method A (Trapezoid Form)}

In Method $A$, the set of detections of cell $n$ for an entire day $\left(k_{n, 1}, f_{n, 1}\right), \ldots,\left(k_{n, j}, f_{n, j}\right), \ldots$, is sorted in an ascending order according to the values of traffic density. After that, we derive a new, sorted set $\left(\mathrm{k}_{\mathrm{n}, \mathrm{i}}, \mathrm{f}_{\mathrm{n}, \mathrm{i}}\right)$ for which $\mathrm{k}_{\mathrm{n}, \mathrm{i}+1} \geq \mathrm{k}_{\mathrm{n}, \mathrm{i}}, \forall \mathrm{i}$. If the measurements of traffic flow, $\mathrm{f}$ (vph), and traffic density, $\mathrm{k}(\mathrm{vpkm})$, are provided for an entire day, then there is a set of density-flow measurements $\left(k_{n, p}, f_{n, p}\right)$ for which:

$$
\mathrm{Q}_{\mathrm{n}}=\mathrm{f}_{\mathrm{n}, \mathrm{p}}=\operatorname{maxf}_{\mathrm{n}, \mathrm{i}}, \quad \forall \in\{1, \ldots . \mathrm{H}\}
$$

The traffic capacity, Qn, is equal to the maximum daily flow and the critical density is the value of density for which the maximum daily flow was observed:

$$
\mathrm{k}_{\mathrm{n}, \mathrm{crit}}=\mathrm{k}_{\mathrm{n}, \mathrm{p}}
$$

The free flow speed (average vehicle speed within a cell measured under low traffic density conditions), $\mathrm{v}(\mathrm{km} / \mathrm{hr})$, is estimated by performing a least-squares fit on the flow.vs.density data in the section where the real density has a lower value than the critical density:

$$
\widetilde{\mathrm{f}_{\mathrm{n}, \mathrm{1}}}=\beta_{0}+\beta_{1} \mathrm{k}_{\mathrm{n}, \mathrm{i}, y^{\prime}} \quad \mathrm{i} \in\{1, \ldots \mathrm{p}\}
$$


The parameters $\beta_{0}, \beta_{1}$ establish the linear relationship between the traffic flow, $f$, and traffic density, $\mathrm{k}$, for $\mathrm{i} \in\{1, \ldots \mathrm{p}\}$ and are extracted from real measurements after performing a least-squares fit. The first point of the line is forced to be the origin of the coordinate plane by setting $\beta_{0}=0$.

The congested speed (average vehicle speed measured under congested conditions), w $(\mathrm{km} / \mathrm{hr})$, is estimated by performing also a least-squares fit on the flow.vs.density data at the section where the real density has a higher value than the critical density $(i=p+1, \ldots . \mathrm{H})$ :

$$
\widetilde{\mathrm{f}_{\mathrm{n}, \mathrm{l}}}=\beta_{0}+\beta_{1} \mathrm{k}_{\mathrm{n}, \mathrm{i},}, \quad \mathrm{i} \in\{\mathrm{p}+1, \ldots . \mathrm{H}\}
$$

The jam density is: $\mathrm{k}_{\mathrm{n}, \mathrm{jam}}=-\frac{\beta 0}{\beta 1}$. Moreover, the congested speed is derived from the equation:

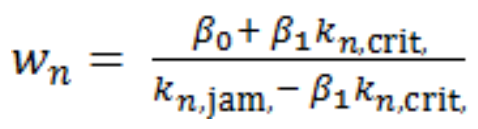

The regression line for the congested speed is shown in Fig. 2. The capacity shows significant drop (> 20\%) from its pre-queue value once congestion settles and bottleneck starts operating at queue-discharge conditions. The instantaneous capacity drop for cell $\mathrm{n}$, $\mathrm{CD}_{\mathrm{n}}$, is given by:

$$
C D_{n}=\frac{Q_{n}-\left(\beta_{0}+\beta_{1} k_{n, \text { crit }}\right)}{Q_{n}} 100 \%
$$

In summary, Method A calibrates the fundamental diagram automatically, and data collection is the only non-automatic stage of this procedure. The following algorithm is provided to summarize the method.

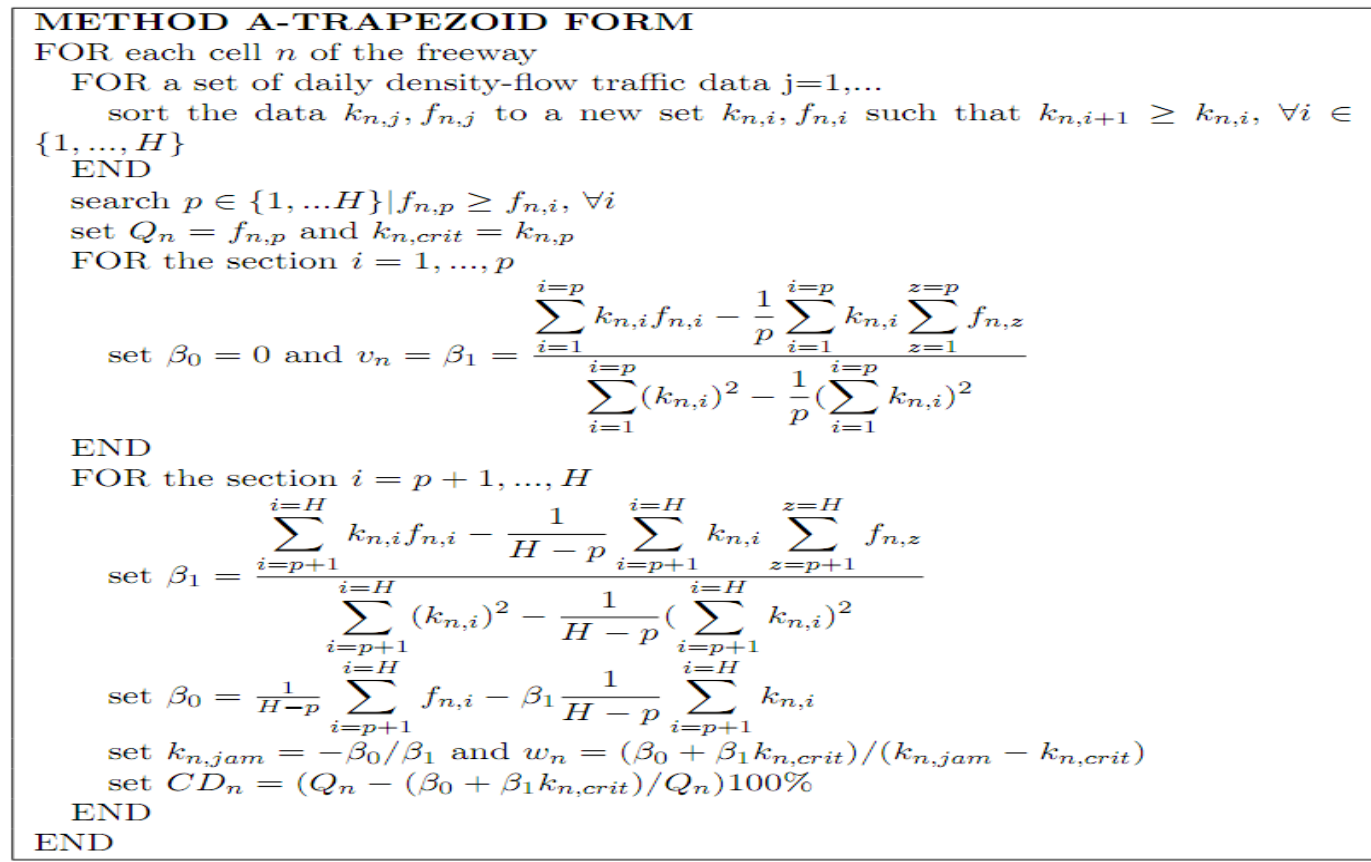




\subsection{Method B - Enforcement of Triangular Constraint}

In Method B, the fundamental diagram adopts a triangular form. First, the capacity of a cell is also set equal to the highest value of daily flow. In addition, a universal value for the critical speed (C.S. $=85 \mathrm{kmph}$ ) is arbitrary introduced as a critical indicator for assessing the traffic conditions within a cell. For each traffic detection, if the observed average speed within a cell is higher than the critical speed, then free flow conditions are assumed and vice versa.

The free flow speed, $\mathrm{v}(\mathrm{km} / \mathrm{hr})$, is estimated by performing a least-squares fit on a subset of flow.vs.density data, at which the measured speed in a cell, $\mathrm{g}_{\mathrm{n}, \mathrm{j}}, \mathrm{i} \in\left\{1_{1}, \mathrm{H}\right\}$ has a higher value than the critical speed (C.S.). On the other hand, the wave speed, $w_{n_{s}}(\mathrm{~km} / \mathrm{hr})$, is estimated by performing a least-squares fit on the section of flow. vs. density data at which the density has a higher value than the new critical density, $\mathrm{k}_{\mathrm{n} \text {,crit: }}$

For each cell $n$ of the freeway we introduce a dummy variable $l_{1}=0$. Then, for a set of density-flow traffic data $\mathrm{i}=1, \ldots . \mathrm{H}$ we set $\mathrm{l}_{1}=\mathrm{l}_{1}+1, \emptyset_{\mathrm{n}}, \mathrm{i}_{2}=\mathrm{f}_{\mathrm{n}, \mathrm{i}}$ and $\mathrm{k}_{\mathrm{n}} \mathrm{l}_{1}=\mathrm{k}_{\mathrm{n}, \mathrm{i}}$ if $\mathrm{g}_{\mathrm{n}, \mathrm{i}}>\mathrm{C}$. S. After completing this step we compute the free flow speed and the critical

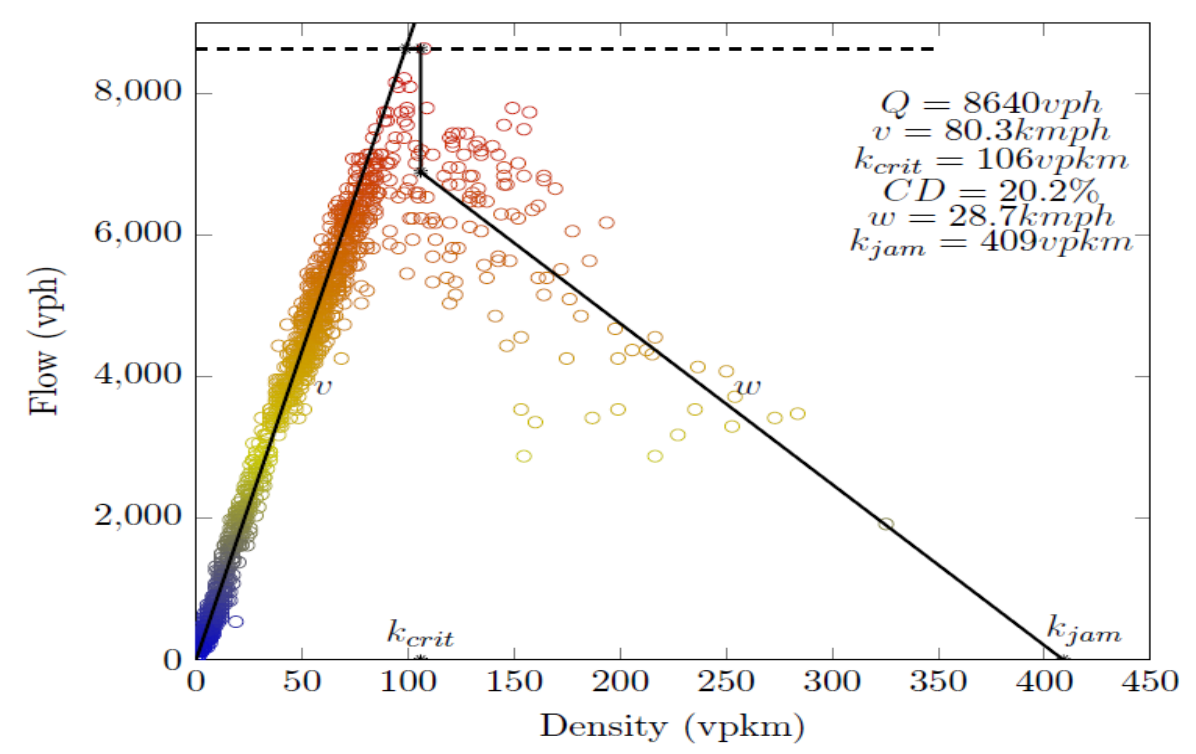

Figure 2. Trapezoid-Shaped Fundamental Diagram with Method A

density as follow:

$$
\begin{gathered}
k_{\text {nerit }}=\frac{f_{n, \mathrm{i}}-\beta_{0}}{\beta_{1}} \\
v_{n}=\frac{Q_{\mathrm{n}}}{k_{\text {nucrit }}}
\end{gathered}
$$

, where:

And

$$
\beta_{0}=\frac{1}{\mathrm{l}_{1}} \sum_{u=1}^{u=\mathrm{l}_{1}} Q_{n u}-\beta_{1} \frac{1}{\mathrm{l}_{1}} \sum_{u=1}^{u=l_{1}} k_{n u}
$$




$$
\beta_{1}=\frac{\sum_{u=1}^{u=\mathbb{I}_{1}} k_{n u, u} Q_{n, u}-\frac{1}{\mathbb{I}_{1}} \sum_{u=1}^{u=\mathbb{I}_{1}} k_{n u} \sum_{z=1}^{z=\mathbb{I}_{1}} Q_{n, z}}{\sum_{u=1}^{u=\mathbb{I}_{1}}\left(k_{n, u}\right)^{2}-\frac{1}{\mathbb{I}_{1}}\left(\sum_{u=1}^{u=\mathbb{1}_{1}} k_{n u u}\right)^{2}}
$$

For the same density-flow dataset we set initially $l_{2}=0$ and we update it to $l_{1}=l_{1}+1$ and set $Q_{n \cdot l_{1}}=f_{n . i}$ and $k_{n \cdot l_{1}}=k_{n . i}$ if $k_{n . i}>k_{n . c r i t}$. The jam density, the congested speed and the capacity drop are given by:

$$
\begin{gathered}
\mathrm{k}_{\mathrm{n}, \mathrm{jam}}=\frac{-\beta_{0}}{\beta_{1}} \\
w_{n}=\frac{\beta_{0}+\beta_{1} k_{\text {n,crit }}}{k_{m, j a m}-k_{\text {m.crit }}} \\
C D_{n}=\left(\frac{Q_{n}-\left(\beta_{0}+\beta_{1} k_{\text {n,crit }}\right)}{Q_{n}}\right) 100 \%
\end{gathered}
$$

, where:

and

$$
\beta_{0}=\frac{1}{\mathbb{1}_{z}} \sum_{u=1}^{u=1_{2}} Q_{n \cdot u}-\beta_{1} \frac{1}{1_{z}} \sum_{u=1}^{u=1_{z}} k_{n, u}
$$

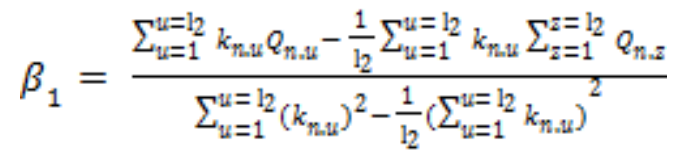

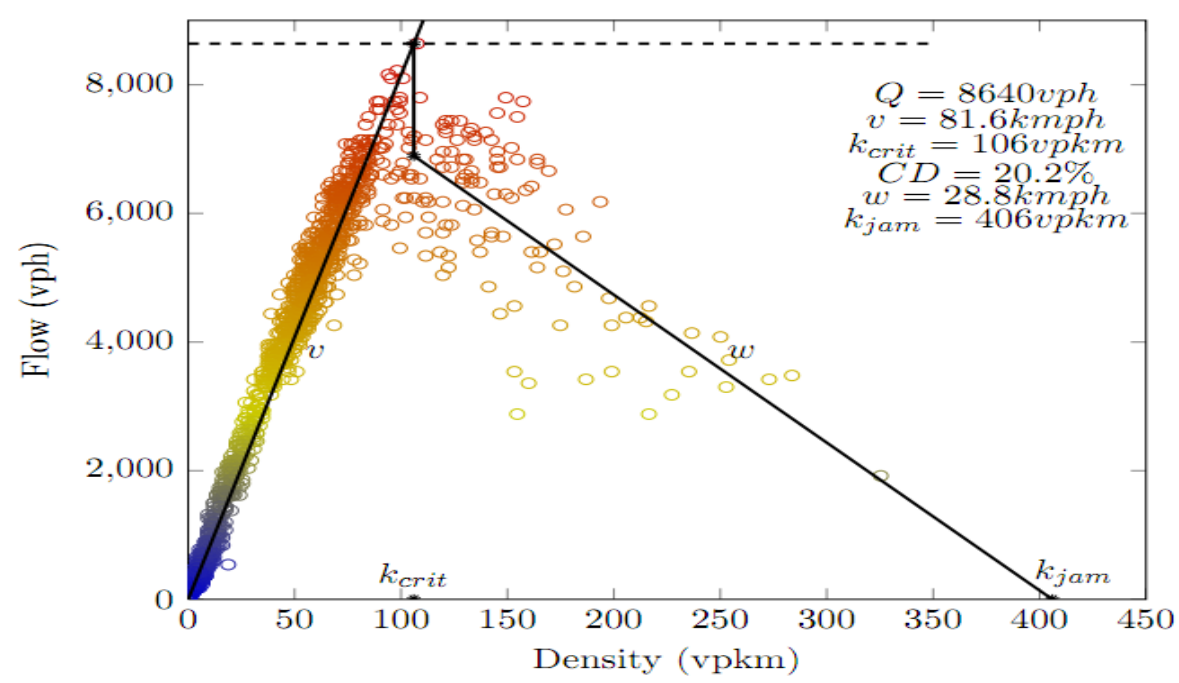

Figure 3. Triangular-Shaped Fundamental Diagram with Method B

\subsection{Method C - Estimation of Congestion Speed with Quantile Regression}

In this calibration method, the capacity of each cell and the free flow speed are estimated in the same way as with Method A. The congested speed though, w, is estimated with the use of quantile regression [15]. 
Method $\mathrm{C}$ projects different values of wave speed, w, jam density, $\mathrm{k}_{\mathrm{jam}}$, and capacity drop, CD, compared with Method A and a comparison between Method A and Method C is provided in Figure 4.

After the computation of kn,crit, the flow density points with density values higher than the critical density are partitioned along the horizontal axis - the density axis - into over-lapping bin cells of 10 data points each. Each bin cell $\mathrm{j}$ embraces ten (10) observations and has a BinDensity, $B_{d}$, which is the mean of the 10 density values in the bin, and a BinFlow, $B_{f}$, which is the largest non-outlier flow value among the 10 flow values in the bin. If $Q_{1}$,

$Q_{3}$ are the 25th and 75th percentiles of the data points in the bin and IQR $=Q_{3}-Q_{1}$, then for each bin cell $\mathrm{j}$ :

$$
\begin{aligned}
B_{d, j}= & \left(\sum_{j, k}^{10}=1 k_{r}\right) / 10 \\
& B_{f, j}=\max \left(f_{r} 1 f_{r}<Q_{3}+1.5 I Q R\right)
\end{aligned}
$$

In this case, instead of having $\mathrm{H}-(\mathrm{p}+1)$ measurements $(\mathrm{kn}, \mathrm{i}, \mathrm{fn}, \mathrm{i})$ with a higher traffic density than the critical one, we consider only $(H-(p+1)) / 10$ measurements $\left(B_{d_{j} \mathrm{j}}, B_{f_{j} j}\right)$. By using those measurements, we perform a least-squares calibration for the estimation of the wave speed and jam density.

$$
\begin{aligned}
\widehat{\mathrm{B}}_{\mathrm{f}, \mathrm{j}} & =\beta_{0}+\beta_{1} \mathrm{~B}_{\mathrm{d}, \mathrm{j}} \\
k_{\mathrm{n}, j a m, j} & =-\beta_{0} / \beta_{1}
\end{aligned}
$$

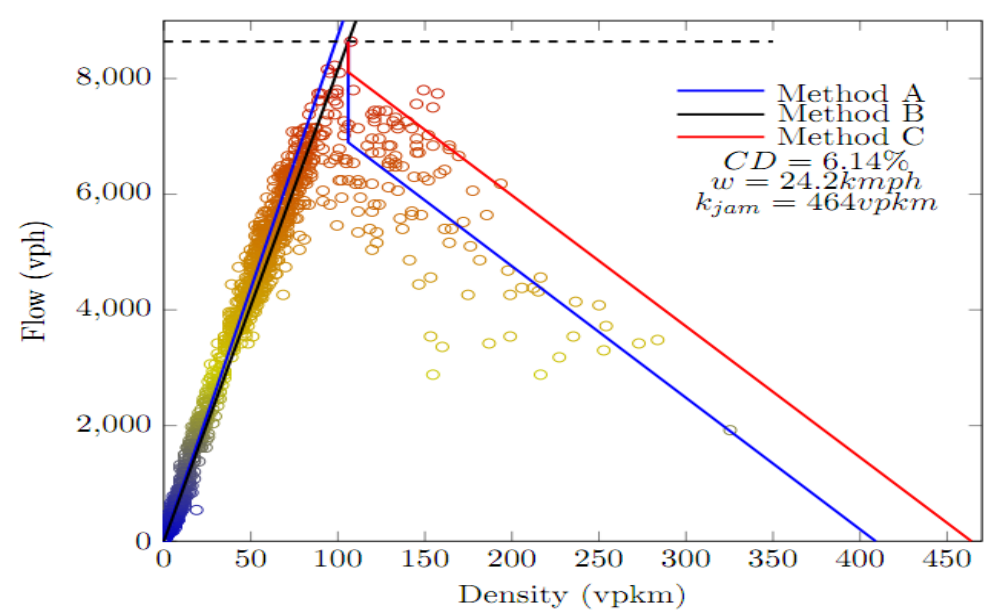

Figure 4. Fundamental Diagram Calibration-Comparison between Methods A, B and $\mathrm{C}$

\subsection{Calibration Performance in Extreme Scenarios}

Before studying the effect of parameters estimation to first-order models, we examine the performance of the three calibration methods in extreme scenarios (i.e., erroneous data observations, datasets with multiple outliers). 
When the quality of traffic data is acceptable, each one of the three calibration methods provide realistic estimations of traffic parameters. Nevertheless, in many cases, either due to missing data or insufficient detections in the congested section of the fundamental diagram, the estimation of traffic parameters is unrealistic. After implementing the three calibration methods in the MIDAS dataset from 16th-20th of May, 2011, we present an example of inadequate parameter estimation with each calibration method in Fig. 5. In addition, Table 2 summarizes the calibration performance of the methods in extreme scenarios (the third column contains the number of calibrated fundamental diagrams for each day without counting the cells with missing data, and in the other three columns we present the number of calibration failures with Methods A, B and C. We assume that a calibration failure occurs either when the calibration predicts a negative value for one or more traffic parameters or when the predicted jam density is $150 \%$ higher than the average jam density estimated by the two other methods.

Table 2. Performance of the Three Calibration Methods in Extreme Cases

\begin{tabular}{|c|c|c|c|c|c|}
\hline Date: May,2011 & No of Detectors & $\begin{array}{c}\text { Cells with } \\
\text { Healthy Data }\end{array}$ & $\begin{array}{c}\text { Method } \\
\text { A/Calibration } \\
\text { Failures }\end{array}$ & $\begin{array}{c}\text { Method } \\
\text { B/Calibration } \\
\text { Failures }\end{array}$ & $\begin{array}{c}\text { Method } \\
\text { C/Calibration } \\
\text { Failures }\end{array}$ \\
\hline 16th & 99 & 71 & 31 & 26 & 48 \\
\hline 17th & 99 & 54 & 7 & 8 & 18 \\
\hline 18th & 99 & 77 & 0 & 1 & 8 \\
\hline 19th & 99 & 85 & 7 & 8 & 18 \\
\hline 20th & 99 & 72 & 14 & 13 & 22 \\
\hline$>\mathrm{D}$ & 495 & 359 & 59 & 56 & 114 \\
\hline \multicolumn{3}{|c|}{ Percentage of Failure } & $16.43 \%$ & $14.77 \%$ & $31.75 \%$ \\
\hline
\end{tabular}

\subsection{Remarks on the Results in Extreme Cases}

The quartile regression, which is used in Method C, 11 provides higher estimations for the value of jam density (in some cases the estimated jam density is $150 \%$ higher than the estimations from the other two methods). This occurs because each bin cell includes ten detections from the congested area; hence, if the number of measurements in the congested area is small, then the values of bin flow are not representative, resulting to unreasonable values of jam density (station 4860 in Figure 5). Moreover, the quantile regression requires more than 30 detections at the congested area and thus its failure rate for estimating the jam density is up to $31.75 \%$.

Methods A and B return similar results regardless their differences during the calibration. Method A yields unrealistic results only in cases of poor data (i.e., absence of traffic data). However, there are some cases (such as station 4959 in Figure 5 ), where the regression line fell below the capacity line, and the fundamental diagram is not trapezoid-shaped since the maximum value of flow (capacity) is not following the pattern of other measurements.

Method B fails to produce a reasonable fundamental diagram in the $14.77 \%$ of cases (Table 2). Nevertheless, most fundamental diagrams do not estimate adequately the free flow speed because the universal free flow speed, $85 \mathrm{kmph}$, is empirically selected and is either higher or lower than the appropriate one. As a result, measurements that belong normally in the congested area are included in the uncongested, and the estimated fundamental diagram reproduces a pattern that does not capture the nature of the measurements. For instance, in station 4909 (Figure 5) the universal free flow speed is too high for that cell and 
measurements from the congested area are included to the free-flow zone, resulting a distorted least-squares fit and an inaccurate estimation of the free flow speed and critical density. The opposite problem is encountered when the universal free flow speed is lower than the appropriate one for a cell.
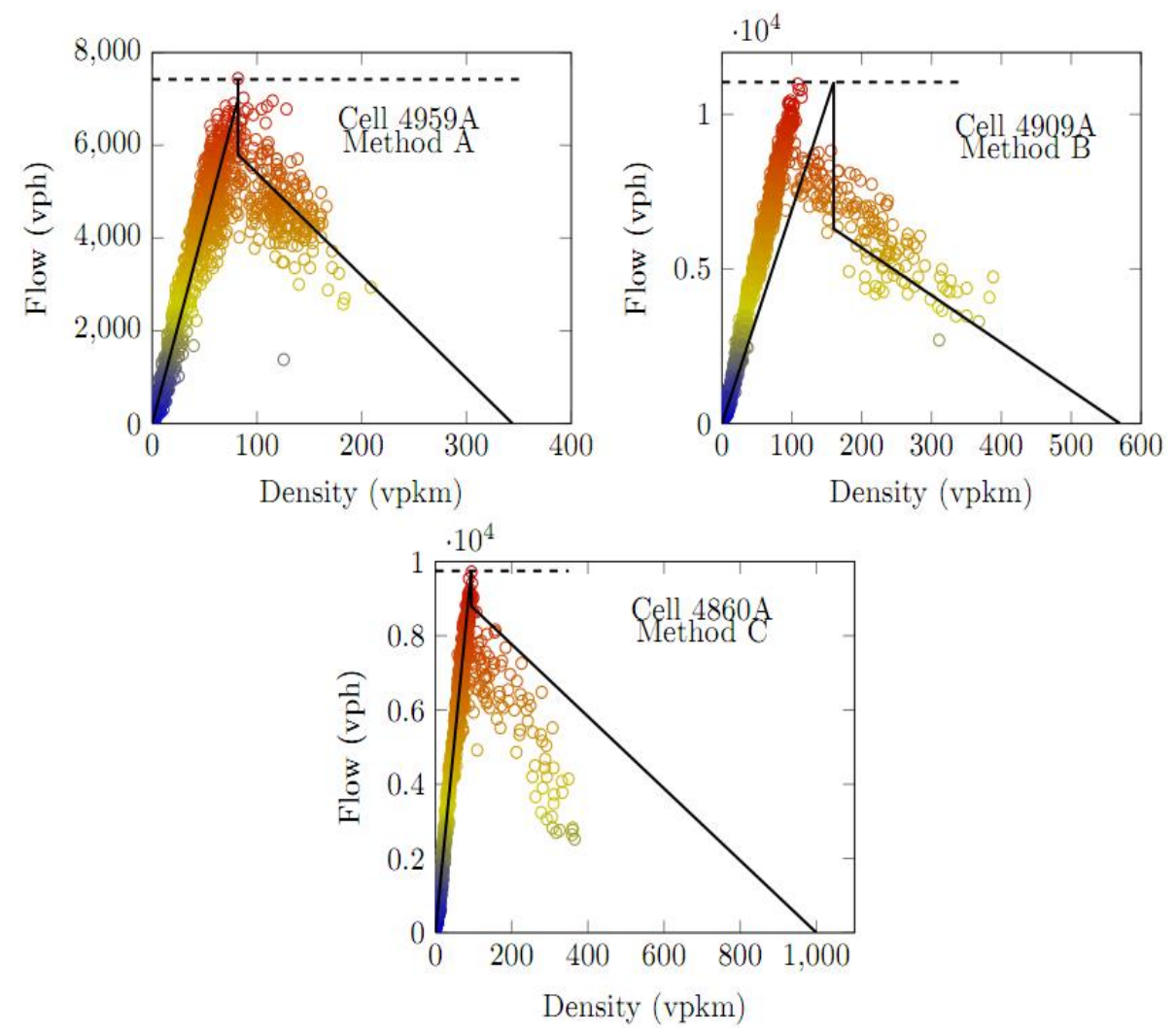

Figure 5. Fundamental Diagram Calibration Results in Extreme Scenarios with Methods A, B and C

\section{Numerical Experiments}

The required traffic data for the estimation of traffic parameters and the implementation of macroscopic models is provided by MIDAS data sets [19] and includes measurements from 99 detectors of motorway M25 (junction 9A to 16). The distance between subsequent detectors is $\mathrm{L}=500 \mathrm{~m}$ and the examined road segment includes 8 on-ramps and 8 off-ramps. Every minute, each detector returns the value of 33 traffic parameters, and thus the daily data from each detector contains 1440 measurements for each parameter. Furthermore, we use the traffic detections from a five-day period (16th, 17th, 18th, 19th and 20th of May 2011) for our case studies. Due to lack of detections only 60 cells out of 99 -including 5 onramps and 6 offramps- will be examined, starting from station 4742A and ending at station 5020A.

In order to provide a comprehensive description of the geometry, the number of lanes, the location of ramps and cells and the characteristics of the provided traffic data Table 3 and Figure 6 are presented below. 
Table 3. M25 Report of Missing Data from May 16th-20th, 2011

\begin{tabular}{|c|c|c|c|c|c|}
\hline Station & Dates & Station & Dates & Station & Dates \\
\hline $4777 \mathrm{~A}$ & 16th,20th & $4871 \mathrm{~A}$ & 16th, 17th & $4945 \mathrm{~A}$ & 16 th \\
\hline $4787 \mathrm{~A}$ & 17 th & $4876 \mathrm{~A}$ & 16 th, 17 th & 4949A & 16 th \\
\hline $4832 \mathrm{~A}$ & 16 th & 4879A & 16th, 17th & $4993 \mathrm{~A}$ & 17 th \\
\hline $4836 \mathrm{~A}$ & 16 th & $4883 \mathrm{~A}$ & 17 th & 4998A & 17 th \\
\hline $4843 \mathrm{~A}$ & 17 th & $4887 \mathrm{~A}$ & 16th, 17th & $5002 \mathrm{~A}$ & 16th, 17th \\
\hline $4848 \mathrm{~A}$ & 16th, 17th & 4898A & 17 th & $5006 \mathrm{~A}$ & 17 th \\
\hline $4854 \mathrm{~A}$ & 16th, 17th & $4903 \mathrm{~A}$ & 16th & $5010 \mathrm{~A}$ & 16th, 17th \\
\hline $4860 \mathrm{~A}$ & 16th, 17th & $4909 \mathrm{~A}$ & 16th & $5015 \mathrm{~A}$ & 16th, 17th,20th \\
\hline $4866 \mathrm{~A}$ & 16th, 17th & $4912 \mathrm{~A}$ & 16th & $5020 \mathrm{~A}$ & 16th, 17th,20th \\
\hline
\end{tabular}

The automatic calibration algorithms and the first-order macroscopic model are implemented on a $2556 \mathrm{MHz}$-processor machine with 1024 Megabytes RAM. In addition, the algorithms are programmed on MATLAB R2007b and the outcomes are the estimation of traffic parameters and the simulation of traffic dynamics for each day. A quantitative and qualitative assessment regarding the conformance of the models to reality is conducted via a series of metrics and figures.

The M25 section from junction 9A "Leatherhead" to 16 "Gerrards Cross" includes 99 links; however, the boundary conditions of macroscopic models require free-flow conditions at the exit of the mainline during the simulation, otherwise the backwave effects (due to the formation and spread of congestion from a road section which does not belong to the area of simulation) are not captured in the simulation. Hence, after a visual inspection of real traffic conditions on M25 on the 16th, 17th, 18th, 19th and 20th of May, 2011 the time-space area of Figure 7 (stations 4742A-5020A; time 01:20pm-08:00pm) was chosen for our analysis:

In Table 4, the traffic flow error1, the traffic density error2, the total vehicle-hours travelled error 3 and the total kilometres-hours travelled error 4 of first-order models that use the three different automatic calibration methods for the 16th, 17th, 18th, 19th and 20th of May are presented (quantitative indicators of performance). In column 2, the number of imputed detections is presented and indicates the number of stations lacking the required data information. In addition, (Figure 8-Figure 9) present a) the real density contour, b) the simulated density contours, c) the simulated delay, d) the simulated VHT and e) the simulated VKT for each day after the implementation of first-order models with the use of calibration different methods $(\mathrm{A}, \mathrm{B}, \mathrm{C})$. These figures provide the basis for qualitative assessment.

$$
\begin{aligned}
& { }^{1} E F=\frac{1}{\left(t_{v}-t_{0}\right) / d t+1} \sum_{t=t_{0}}^{t=t_{v}} \frac{1}{N} \sum_{n=1}^{n=N} \frac{\left|f_{n}(t)-\widehat{f}_{n}(t)\right|}{f_{n}(t)} \\
& { }^{2} E D=\frac{1}{\left(t_{v}-t_{0}\right) / d t+1} \sum_{t=t_{0}}^{t=t_{v}} \frac{1}{N} \sum_{n=1}^{n=N} \frac{\left|k_{n}(t)-\widehat{k}_{n}(t)\right|}{k_{n}(t)} \\
& { }^{3} T V H T=\frac{1}{\left(t_{v}-t_{0}\right) / d t+1} \sum_{t=t_{0}}^{t=t_{v}}\left|\frac{\sum_{n=1}^{n=N} k_{n}(t) \Delta x_{n} d t-\sum_{n=1}^{n=N} \widehat{k}_{n}(t) \Delta x_{n} d t}{\sum_{n=1}^{n=N} k_{n}(t) \Delta x_{n} d t}\right| \\
& { }^{4} T V K T=\frac{1}{\left(t_{v}-t_{0}\right) / d t+1} \sum_{t=t_{0}}^{t=t_{v}}\left|\frac{\sum_{n=1}^{n=N} k_{n}(t) v_{n}(t) \Delta x_{n} d t-\sum_{n=1}^{n=N} \hat{k}_{n}(t) v_{n}(t) \Delta x_{n} d t}{\sum_{n=1}^{n=N} k_{n}(t) v_{n}(t) \Delta x_{n} d t}\right|
\end{aligned}
$$




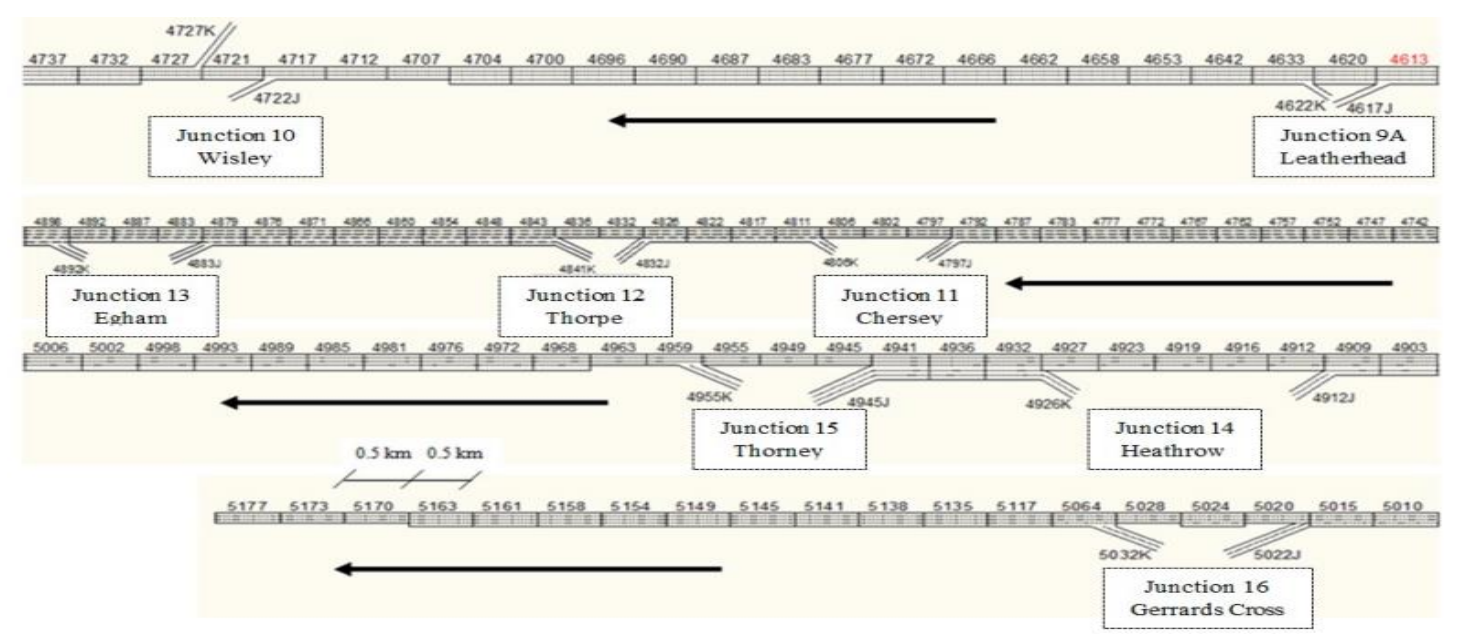

Figure 6. M25 Graphic Representation from Station 4613A to 5117A

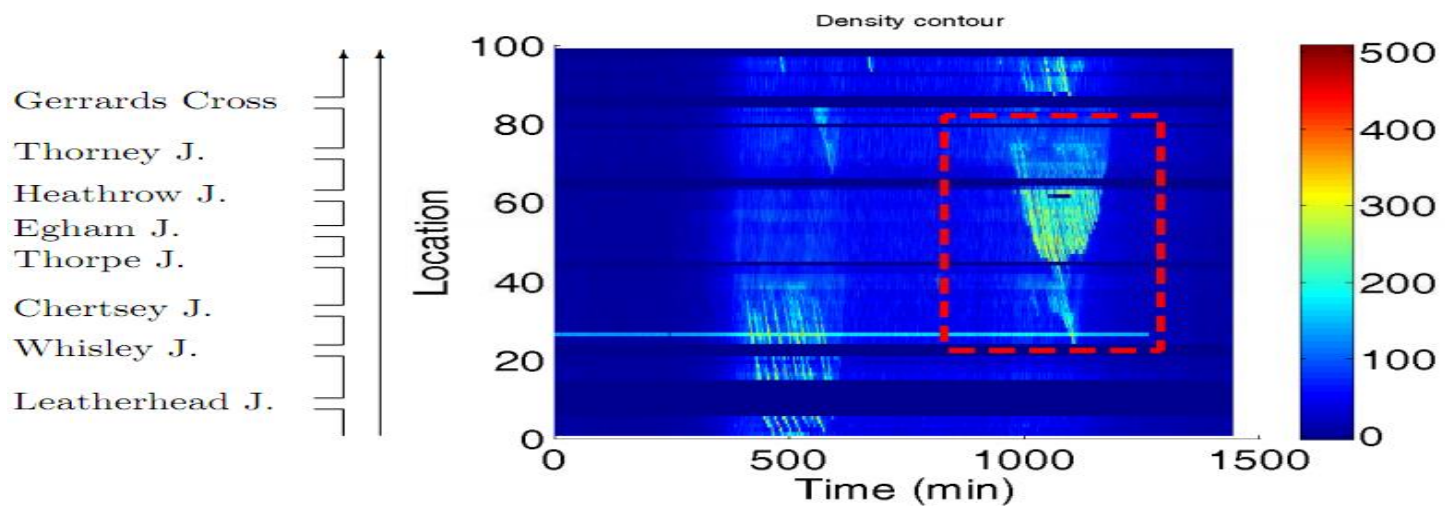

Figure 7. Density Contour: the examined area lies within the red rectangle (18th of May, 2011)

Table 4. Summary of First-Order Models' Performance Subject to the Use of Different Calibration Methods General Characteristics: Number of Cells (60); Time (400min); Simulation Steps (1600)

\begin{tabular}{|l|r|r|r|r|r|r|r|}
\hline \multicolumn{6}{|c|}{ General Characteristics: Number of Cells (60); Time (400min); Simulation Steps (1600) } \\
\hline Date & $\begin{array}{l}\text { Imputed } \\
\text { Detections }\end{array}$ & $\begin{array}{l}\text { Calibration } \\
\text { Method }\end{array}$ & $\begin{array}{l}\text { Flow Error, } \\
\text { EF\% }\end{array}$ & $\begin{array}{l}\text { Density Error, } \\
\text { ED\% }\end{array}$ & $\begin{array}{l}\text { TVHT } \\
\text { Error\% }\end{array}$ & $\begin{array}{l}\text { TVKT } \\
\text { Error\% }\end{array}$ \\
\hline 17th of May & $20 / 60$ & Method A & 12.6 & 13.75 & 21 & 16 \\
\hline & & Method B & 12.6 & 13.01 & 22 & 16 \\
\hline & & Method C & 12.6 & 12.80 & 30 & 16 \\
\hline 18th of May & $3 / 60$ & Method A & 12.1 & 9.22 & 18 & \\
\hline & & Method B & 12.1 & 9.20 & 20 & \\
\hline & & Method C & 12.2 & 9.34 & 17 & \\
\hline 19th of May & $9 / 60$ & Method A & 15.8 & 12.96 & 12 & 10 \\
\hline & & Method B & 15.9 & 13.05 & 17 & 11 \\
\hline
\end{tabular}




\begin{tabular}{|l|r|r|r|r|r|r|}
\hline & & Method C & 15.9 & 13.03 & 12 & 10 \\
\hline 20th of May & $12 / 60$ & Method A & 14.8 & 12.05 & 14 & \\
\hline & & Method B & 14.7 & 12.27 & 16 & \\
\hline & & Method C & 14.8 & 12.00 & 14 & \\
\hline & & & & & & \\
\hline
\end{tabular}

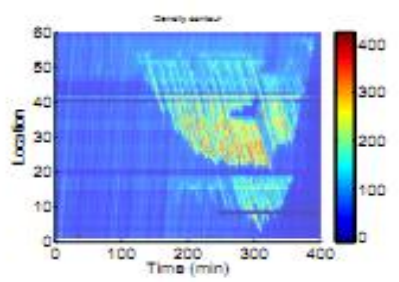

(a) Observed Density
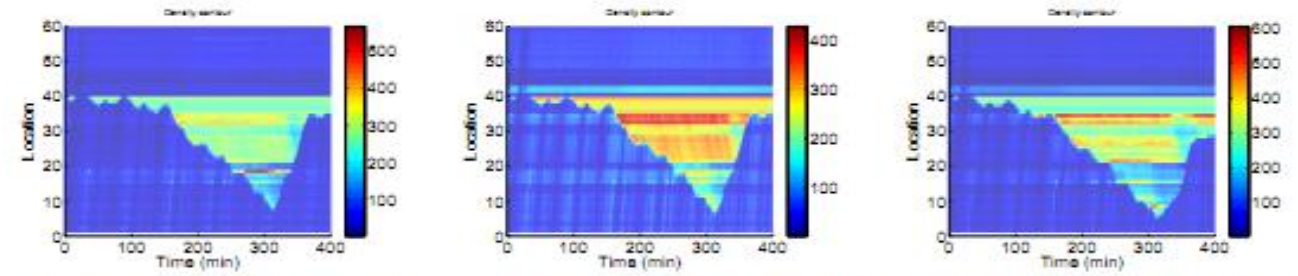

(b) Simulated Density (Method A)

B)

(d) Simulated Density (Method

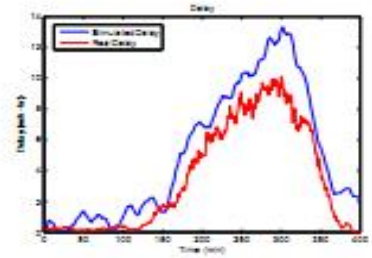

(e) Delay (Method A)

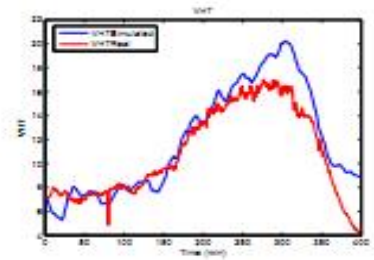

(h) VHT (Method A)

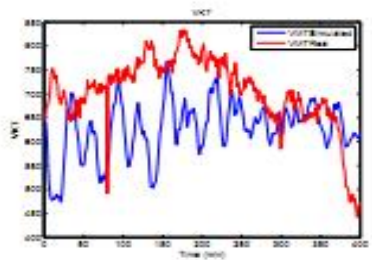

(k) VKT (Method A)

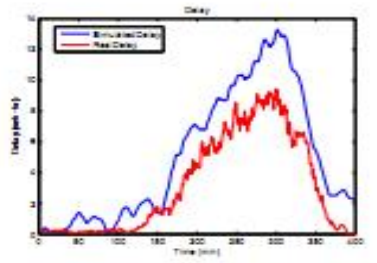

(f) Delay (Method B)

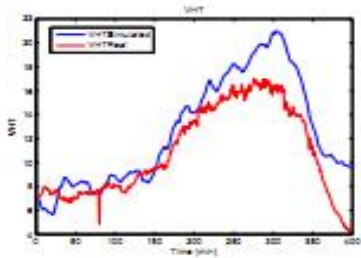

(i) VHT (Method B)

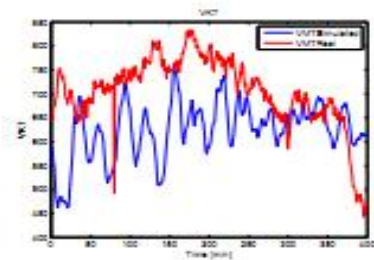

(1) VKT (Method B)

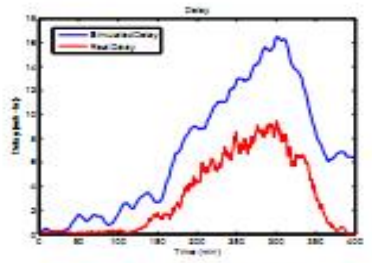

(g) Delay (Method C)

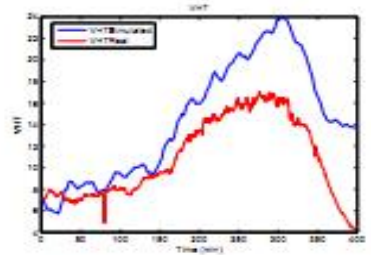

(j) VHT (Method C)

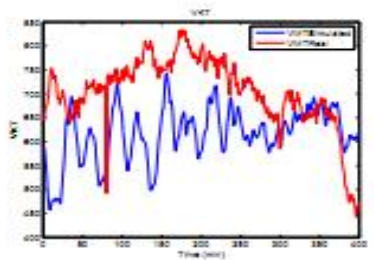

(m) VKT (Method C)

Figure 8. Macrosimulation Results with the Use of Different Fundamental Diagrams: May 19th, 2011 


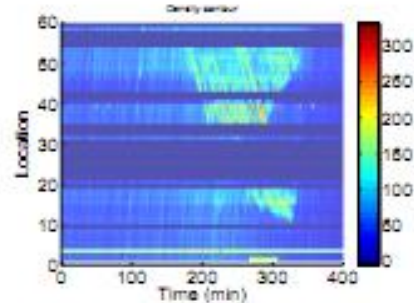

(a) 17th May, Observed Density

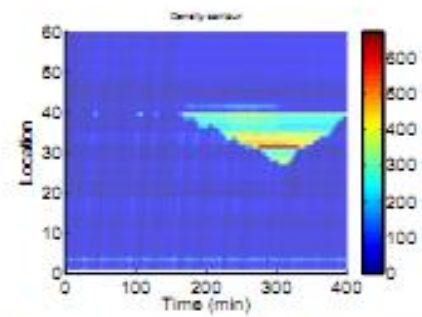

(d) 17th May, Simulated Density (Method C)

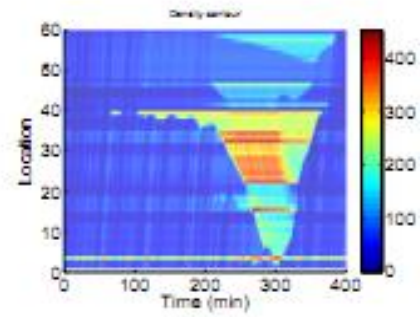
(g)

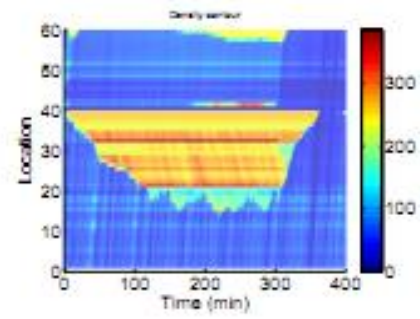

(j) 20th May, Simulated Density (k) 20th May, Simulated Density (Method A)

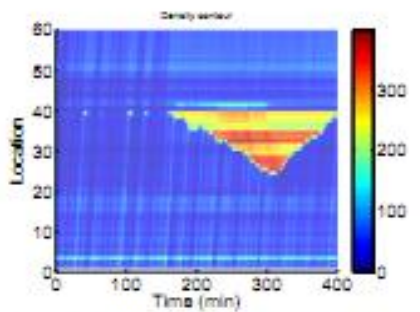

(b) 17th May, Simulated Density (Method A)

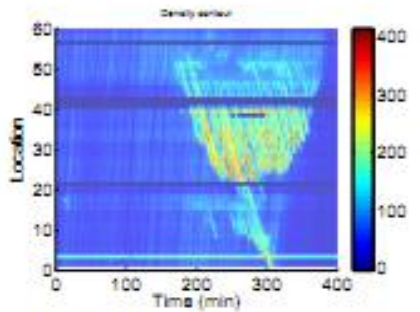

(e) 18th May, Observed Density

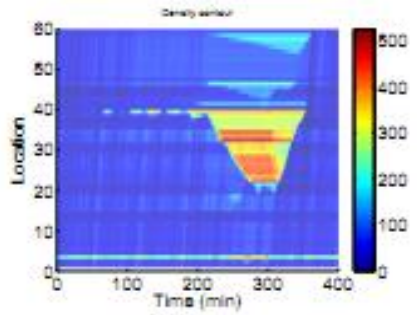

(Method C)

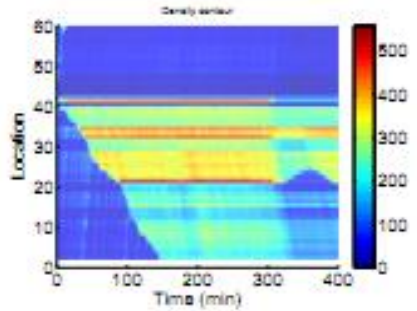

(Method B)

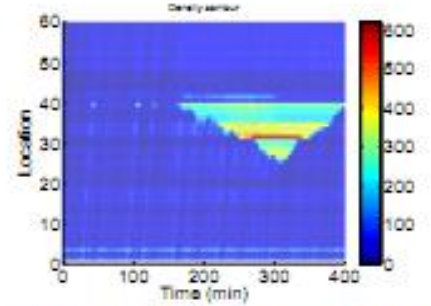

(c) 17th May, Simulated Density (Method B)

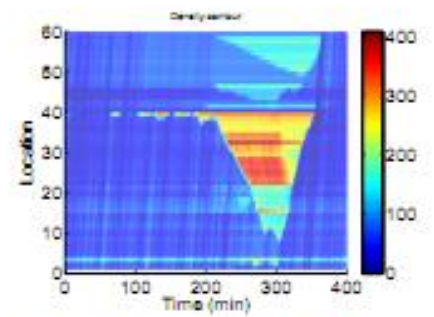

(f) 18th May, Simulated Density (Method A)

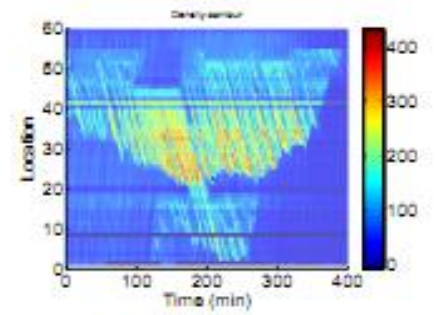

(i) 20th May, Observed Density

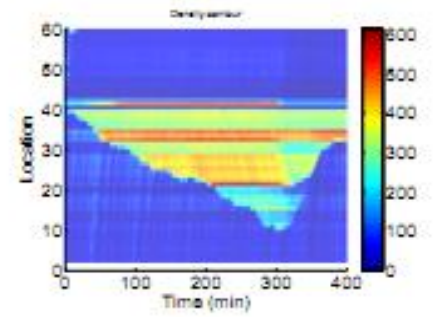

(1) 20th May, Simulated Density (Method C)

\section{Figure 9. Observed and Estimated Densities with Different Fundamental Diagrams}

\section{Conclusion}

In this study, we introduced three methods for the calibration of the fundamental dia-gram that allow an instantaneous capacity drop after shifting from free flow to congested conditions. Firstly, we examined: a) their performance in extreme scenarios (Sections 3.43.5 ), and b) their influence to the performance of first-order macroscopic models by 
simulating the traffic flow dynamics on motorway M25 from junction 11A (Chertsey J.) to 16A (Gerrards Cross) and from 1:20 p.m. to 08:00 p.m. over a week's period.

The quantitative results from the simulations are presented in Table 4 (from 16th-20th of May) and the qualitative results in Fig. 8, Fig. 9. Among those 5 days, the 16th of May was not examined due to the lack of data which inhibits any attempt for traffic parameters estimation. After performing both quantitative and qualitative analysis, we provide below a summary of the key findings from the simulations regarding the significance of calibration methods to first-order models:

The modified first-order models simulated the evolution of traffic with a $12-16 \%$ traf-fic flow error, 9-13\% traffic density error, $12-31 \%$ TVHT error and 7-17\% TVMT error by using different traffic parameter values according to the applied calibration method. After the simulation, the deviation of traffic flow errors with different cali-bration methods was less than $0.15 \%$, the deviation of traffic density errors was less than $0.27 \%$, and less than $0.35 \%$ for TVMT errors. Therefore, the calibration of the fundamental diagram is not affecting significantly -at least quantitatively- the performance of first-order models.

TVHT errors are an exception with a variance of simulation results with different calibration methods up to $8.7 \%$.

Unlike the quantitative performance, the qualitative performance of first-order models is significantly affected by the use of different calibration methods. Especially cali-bration Method $\mathrm{C}$ yields in many cases unexpected qualitative results which do not conform to reality and cannot capture accurately the formation of shockwaves(Fig. 9).

The performance of different calibration methods under extreme scenarios varies significantly (in Table 2. Only the $68.25 \%$ of fundamental diagrams were calibrated with Method C, instead of $83.57 \%$ and $85.23 \%$ with Methods A and B).

The results of the study provide evidence that different calibration methods lead to significantly different calibration results in extreme scenarios (i.e., calibration with insufficient data). Moreover, different calibration methods affect the performance of first-order mod-els and more specifically the accuracy of capturing the flow-density evolution from cell to cell (see qualitative results) since the traffic flow evolution from cell to cell is interpreted differently.

On the other hand, the aggregated quantitative metrics (traffic flow, traffic density, TVMT and TVHT errors) proved that the influence of different calibration methods on the overall simulation errors is rather low. This could be occurred either because calibration techniques contribute only partially to the amplification of simulation errors or because the simulation results are influenced by other factors (accumulated errors in the geometry of the network and the length of the cells, empirically imputation of missing data for the cause of the simulation, data absence in secondary roads or in/out flow from/to ramps).

To conclude, different calibration methods make little difference to the overall traffic simulation errors, but they affect significantly the description of flow evolution from cell to cell and the time-space representation of shockwave formations. Simulation results demonstrated also that the performance of first-order models is extremely vulnerable to slight changes on ramp flows (case of missing data on ramp 4841K). Finally, a more detailed study on the significance of missing/imputed on/off-ramp flows and another study on the significance fundamental diagrams to the performance of second or higher order models are proposed as future scientific topics. 


\section{Acknowledgements}

We are thankful to the UK Highway Agency and the UK Department for Transport for providing real time traffic data through MIDAS data sets. The contents of the paper do not reflect the official views of Highway agency and the authors are responsible for the data presented herein.

\section{References}

[1] H. K. Lo, "A cell-based traffic control formulation: Strategies and benefits of dynamic timing plans", Transportation Science, vol. 35, no. 2, (2001) May, pp. 148-164.

[2] T. Schwerdtfeger, "Dynemo: A model for the simulation of traffic flow in motorway net-works", Proceedings of the 9th international Symposium on Transportation and Traffic Theory, pp. 65-87, (1984).

[3] M. Cremer and J. Ludwig, "A fast simulation model for traffic flow on the basis of boolean operations", Mathematics and Computers in Simulation, vol. 28, no. 4, (1986) August, pp. 297-303.

[4] K. Nagel and M. Schreckenberg, "A cellular automaton model for freeway traffic", Journal de Physique I, vol. 2, no. 12, (1992) December, pp. 2221-2229.

[5] R. Wiedemann, "Modelling Of Rti-Elements On Multi-Lane Roads", vol. II, (1991).

[6] M. J. Lighthill and G. B. Whitham, "On kinematic waves. II. a theory of traffic flow on long crowded roads", Proceedings of the Royal Society of London. Series A. Mathe-matical and Physical Sciences, vol. 229, no. 1178, (1955) May, pp. 317-345.

[7] C. F. Daganzo, "The cell transmission model: A dynamic representation of highway traffic consistent with the hydrodynamic theory", Transportation Research Part B: Methodological, vol. 28, no. 4, (1994) August, pp. 269-287.

[8] A. A. Kurzhanskiy and P. Varaiya, "Active traffic management on road net-works: a macroscopic approach", Philosophical Transactions of the Royal Society A: Mathematical, Physical and Engineering Sciences, vol. 368, no. 1928, (2010) October, pp. 4607-4626.

[9] W.-H. Lin and H. K. Lo, "A theoretical probe of a german experiment on stationary moving traffic jams", Transportation Research Part B: Methodological, vol. 37, no. 3, (2003) March, pp. 251-261.

[10] B. G. Heydecker and J. D. Addison, "Analysis and modelling of traffic flow under variable speed limits", Transportation Research Part C: Emerging Technologies, vol. 19, no. 2, (2011) April, pp. 206-217.

[11] M. Carey and M. Bowers, "A review of properties of FlowDensity functions", Transport Reviews, vol. 32, no. 1, (2012), pp. 49-73.

[12] L. Munoz, X. Sun, R. Horowitz and L. Alvarez, "Piecewise-linearized cell transmission model and parameter calibration methodology", Transportation Re-search Record: Journal of the Transportation Research Board, vol. 1965, no. 1, (2006) January, pp. 183-191.

[13] B. S. Kerner, "Experimental features of self-organization in traffic flow", Physical Review Letters, vol. 81, no. 17, (1998) October, pp. 3797-3800.

[14] G. Orosz, R. Eddie Wilson, R. Szalai and G. Stpn, "Exciting traffic jams: Nonlinear phenomena behind traffic jam formation on highways", Physical Review E, vol. 80, no. 4, (2009) October, pp. 046205.

[15] G. Gomes, J. Kwon, G. Dervisoglu, R. Horowitz and P. Varaiya, "Automatic calibration of the fundamental diagram and empirical observations on capacity", Transportation Research Board, 88th Annual Meeting, (2009), pp. 183-191.

[16] G. Muralidharan, A. Dervisoglu and R. Horowitz, "Probabilistic graphical models of fun-damental diagram parameters for freeway traffic simulations", Transportation Research Record, In press.

[17] Transport Research Laboratory. (trl, 2012) transyt 14, July 2012. Accessed: 2012-06-30.

[18] A. Gning, L. Mihaylova and R. K. Boel, "Interval macroscopic models for traffic net-works", IEEE Transactions on Intelligent Transportation Systems, vol. 12, no. 2, (2011), pp. 523-536.

[19] Highways Agency. UK Department for Transport. Midas: Uk road traffic data, July 2012. Accessed: 201206-30. 
International Journal of Transportation

Vol. 2, No. 2, (2014) 